\title{
REMINERALIZATION EFFECT OF POLYASPARTIC ACID IN THE POLYMER-INDUCED LIQUID PRECURSOR PROCESS ON THE INTRAFIBRILLAR DENTIN (SCANNING ELECTRON MICROSCOPE AND ENERGY DISPERSIVE X-RAY ANALYSIS)
}

\author{
RIVANTI IRMADELA DEVINA ${ }^{1}$, ADELINE CLARISSA ${ }^{1}$, NILAKESUMA DJAUHARIE ${ }^{2 *}$, KAMIZAR NAZAR $^{2}$, \\ RATNA MEIDYAWATI ${ }^{2}$
}

${ }^{1}$ Conservative Dentistry Residency Program, Faculty of Dentistry, Universitas Indonesia, Jakarta, Indonesia. ${ }^{2}$ Department Conservative Dentistry, Faculty of Dentistry, Universitas Indonesia, Jakarta, Indonesia. Email: nila.setyopurnomo@gmail.com

Received: 30 July 2019, Revised and Accepted: 01 June 2020

\section{ABSTRACT}

Objective: This study aims to analyze remineralization that occurred in demineralized dentin following polyaspartic acid (pAsp) in PILP immersion.

Methods: Sixteen dentin block samples were immersed in demineralized solution. The samples were divided into controls (no pAsp immersion) or pAsp immersion for 3, 7, or 14 days (n's=4). The samples were evaluated using a scanning electron microscope (SEM) to observe morphology and energy-dispersive X-ray (EDX) to observe calcium and phosphate levels.

Results: SEM revealed that pAsp immersion resulted in significantly higher dentin remineralization compared to the control group. However, there were no significant differences among 3, 7, and 14 days of immersion. EDX revealed that remineralization occurred by the deposition of calcium and phosphate ions.

Conclusion: Including pAsp in the PILP process produced rapid remineralization of demineralized dentin.

Keywords: Intrafibrillar remineralization, Non-collagenous protein analog, Polyaspartic acid, Polymer-induced liquid precursor, Demineralized dentin.

(c) 2020 The Authors. Published by Innovare Academic Sciences Pvt Ltd. This is an open access article under the CC BY license (http://creativecommons. org/licenses/by/4. 0/) DOI: http://dx.doi.org/10.22159/ijap.2020.v12s2.OP-46

\section{INTRODUCTION}

Dentin remineralization can occur conventionally and by guided tissue remineralization. Conventional remineralization works epitaxially by utilizing existing apatite crystals and crystals exclusively deposited on extrafibrillar collagen [1]. Other forms of remineralization do not rely on apatite crystals and can occur in the intrafibrillar matrix, including biomimetic, bottom-up, and GTR [2]. Intrafibrillar remineralization is necessary to improve dentin mechanical properties; the process needs non-collagen proteins (ex. DMP1) to maintain the amorphous calcium phosphate (ACP) form on a nanoscale so that calcium and phosphate ions can penetrate the intrafibrillar collagen to promote biomimetic remineralization [3,4]. However, dental caries and the demineralization process can damage DMP1. Therefore, an analog material to replace DMP1 is necessary for the remineralization process [5].

The polymer-induced liquid precursor (PILP) is currently being developed for biomimetic dentin remineralization [6,7]. PILP uses synthetic polymer materials such as polyaspartic acid (pAsp) that can replace non-collagen proteins in the intrafibrillar collagen dentin remineralization process [8]. In its liquid form, PILP is advantageous because it forms nanodroplets with a diameter of $15-30 \mathrm{~nm}$. This allows for better ACP diffusion into collagen and apatite crystals to quickly fill the gap zone on the intrafibrillar matrix [6]. Several studies that used pAsp analogs in PILP showed significantly better remineralization levels compared to other polymeric materials, such as polyacrylate acid and polyvinyl phosphoric acid $[2,9,10]$. Measuring horse tendons using transmission electron microscopy analysis, Nudelman et al. verified that apatite mineral deposits formed on the intrafibrillar matrix after $72 \mathrm{~h}$ [5]. Burwell et al. demonstrated that affected dentin tissues treated by PILP containing pAsp formed apatite minerals 7 days following treatment and collagen fibrils were fully filled with apatite minerals 14 days following treatment [11].

To date, no studies have observed the effectiveness of PILP containing pAsp in human teeth over a time course of immersion. The current study examined changes in mineral deposits in dentin intrafibrillar collagen following 3, 7, and 14 days of immersion in PILP remineralization solutions that contained pAsp. We hypothesized that this would result in faster remineralization of dentin intrafibrillar collagen compared to the previous studies.

\section{METHODS}

This research was conducted from October to December 2018 at the Dental Material Laboratory of Faculty of Dentistry of Universitas Indonesia and BATAN Laboratory. All the ethical permissions associated in this research were granted by the Ethical Commission of Faculty of Dentistry of Universitas, Indonesia with the protocol number of 050030119. The inclusion criteria are freshly extracted premolar teeth that have no caries, fracture, and no restoration history. Following extraction, teeth were immediately stored in a deionized solution at $4^{\circ} \mathrm{C}$. The teeth were made with dentin blocks $(4.5 \times 4.5 \times 2 \mathrm{~mm}$ in size $)$, which were prepared from the mid-coronal region of the teeth perpendicular to the direction of the dentinal tubules. All of the specimen surfaces were made coarse with $\mathrm{SiC}$ abrasive paper (320-1200 grit), then polished with aqueous diamond suspension (particle sizes: 6.0, 3.0, 1.0, and $0.25 \mu \mathrm{m}$ ). The specimen surface was coated with nail polish varnish except in the opened area $(2.5 \times 2.5 \mathrm{~mm})$.

A total of 16 samples were evenly divided into four groups (n's=4). One group was not immersed in the remineralization solution, whereas the other groups were immersed on days 3,7 , or 14 . This study followed the 
protocol established by Burwell et al. [11]. All samples were immersed and soaked for $66 \mathrm{~h}$ in a demineralization solution $(0.05 \mathrm{M}$ acetate buffer, $2.2 \mathrm{mM}$ calcium phosphate, adjusted $\mathrm{pH}=5.0$ ). After an artificial carious lesion was formed, samples were rinsed with distilled water.

With the exception of Group 1, the samples were immersed in a remineralization solution. The solution included $40 \mathrm{mg}$ of pAsp (Alamanda Polymers Inc, Alabama, USA) with a molecular weight of $23 \mathrm{kDa}, 50 \mathrm{mM}$ TBS with $0.9 \% \mathrm{NaCl}, 0.02 \% \mathrm{NaN}_{3}, 4.5 \mathrm{mM} \mathrm{CaCl}_{3}$, and $2.1 \mathrm{mM} \mathrm{K}_{2} \mathrm{HPO}_{4}$. Specimens were incubated at $37^{\circ} \mathrm{C}$ with continuous shaking for 3,7 , or 14 days.

A scanning electron microscope (SEM) was used to evaluate dentin surface morphology. SEM data were obtained by manual counting and calculated as a percentage using the equation: ([area of dentin tubules containing minerals/total dentin tubule area] $\times 100$ ). The results of this calculation were converted into percentages by multiplying by 100 . Energy-dispersive X-ray (EDX) was used to observe mineral deposits that occur in the form of calcium and phosphate ions on the dentin surface.

Quantitative data were statistically analyzed using SPSS version 22.0 (IBM Statistics for Windows, Version 22.0. Armonk, NY:IBM Corp.). The results were assessed by three observers to examine the interobserver agreement. The reliability of the observer's assessments was tested using Cohen's Kappa test with a minimum reliability level of $80 \%$. Data were then analyzed using a one-way ANOVA with a length of remineralization solution exposure as the independent variable. Bonferroni post hoc tests were run when there were significant differences. The significance level was set as $\mathrm{p}<0.05$.

\section{RESULTS AND DISCUSSION}

SEM and EDX were used to analyze the levels of remineralization induced by PILP containing pAsp that occurred in demineralized dentin over 3, 7, and 14 days of exposure. Dentin morphology observed using SEM is shown in Fig. 1 and Table 1.

Open dentinal tubules surrounded by collagen, indicating a loss of mineral apatite, was observed in demineralized samples (Fig. 1a). Minerals in the demineralized dentin were not entirely lost or dissolved due to calcium phosphate ions obtained from saliva. The demineralization process employed here is analogous to the formation of artificial carious lesions in affected dentin. In affected dentin, collagen denaturation is reversible and allows for remineralization to occur. The remineralization process requires collagen crossing, which is present in affected dentin [12].

A one-way ANOVA analyzing percent of dentinal tubule area that contained minerals revealed a significant effect between groups ( $\mathrm{F}$ value $=11.956$ and $\mathrm{p}=0.001$ ). Bonferroni post hoc analyses revealed that remineralization for 3,7 , and 14 days significantly rescued mineralization levels compared to no treatment $\left(p^{\prime} s<0.001\right)$. There were no significant differences in remineralization between pAsp-treated groups ( $p$ 's $>0.05$ ). These results indicate that remineralization can be induced by PILP containing pAsp with as few as 3 days of treatment.

The current finding is in line with the previous studies. Nudelman et al. (2010) indicated that PILP containing pAsp increases the remineralization capability in the collagen fibrils after $72 \mathrm{~h}$ studied in the horse tendon [5]. The current observation that significant levels of remineralization occur following 3 days of immersion can improve the time efficiency for the treatment of deep carious lesions in patients. According to Xu et al. (2018), calcium and phosphate ions occur after just 30 min of pAsp immersion [13]. However, Burwell et al. (2012) stated that affected dentin can undergo intrafibrillar remineralization with 7 days of pAsp treatment. Furthermore, better intrafibrillar remineralization form was observed at 14 days, which was indicated by full apatite mineral deposition in collagen fibrils [11].

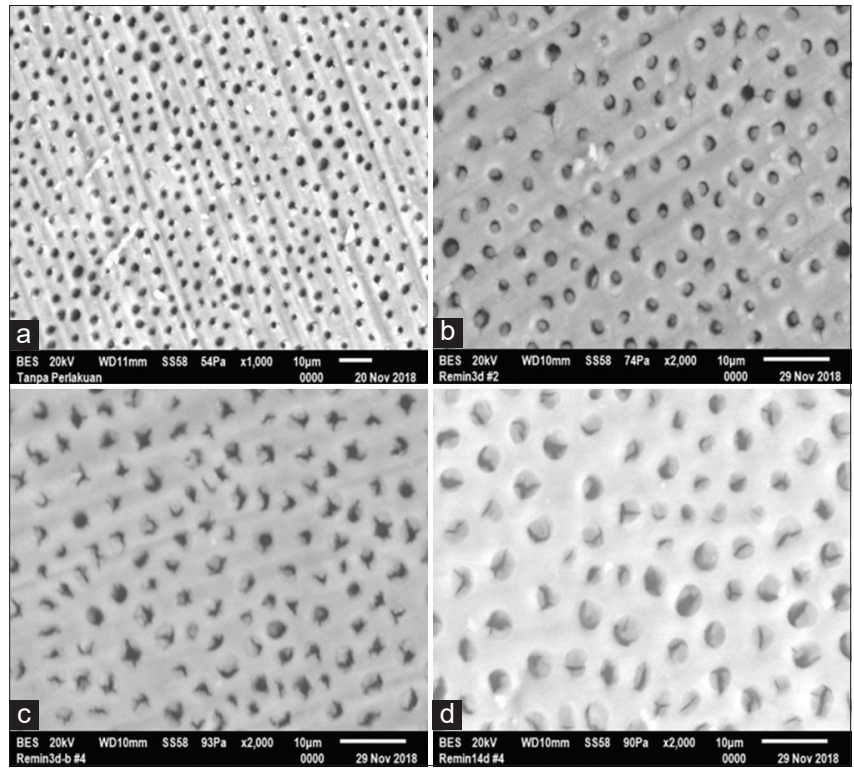

Fig. 1: Scanning electron microscope images of dentinal surface morphology. (a) Demineralized dentin, (b) 3 days pAsp immersion, (c) 7 days pAsp immersion, (d) 14 days pAsp immersion

Table 1: Mean percentage of remineralized dentinal tubules observed in scanning electron microscope images

\begin{tabular}{ll}
\hline Treatment group & Mean \pm SD percentage (\%) \\
\hline Demineralized dentin & $40.35 \pm 30.81$ \\
3 days pAsp immersion & $90.25 \pm 3.807^{* *}$ \\
7 days pAsp immersion & $94.53 \pm 4.464^{* *}$ \\
14 days pAsp immersion & $98.63 \pm 1.981^{* *}$ \\
\hline
\end{tabular}

One-way ANOVA test, significance value $\mathrm{p}<0.05$. Asterisks $\left({ }^{* *}\right)$ indicate different than demineralized dentin group at $\mathrm{p}<0.01$. pAsp: Polyaspartic acid

The remineralization solution is advantageous because, in its liquid form, PILP forms a 15-30 nm diameter nanodroplet that allows quick and efficient ACP diffusion into collagen. ACP then undergoes mineralization into apatite crystals that fill the gap zone on the intrafibrillar matrix [12]. PILP has a negatively charged carboxyl group that binds to positively charged calcium ions. Nudelman et al. (2010) demonstrated that PILP could bind $\times 16$ more calcium than remineralization materials that do not include polymer [5].

Recent work by Bacino et al. (2018) that integrates PILP and restorative dental material stated that the shrinkage of the lesion was reduced after pAsp was added to RM-GI cement. The demineralized dentin area recovered substantially, indicating that pAsp induced remineralization [14]. Similarly, the current study demonstrated that PILP able to remineralize demineralized dentin after immersing with pAsp solution.

EDX was used to observe calcium and phosphate levels which are hydroxyapatite minerals expected to form after remineralization. Ion levels are presented in Fig. 2, whereas the percentage of calcium and phosphate ions is shown in Table 2. This study used EDX to demonstrate that mineral deposits occurred in the form of calcium and phosphate ions in all treatment groups. This indicates that remineralization occurs in the form of calcium and phosphate ion minerals. However, the levels of calcium and phosphate before and after treatment cannot be differentiated clearly because the EDX method does not quantify the baseline level. 


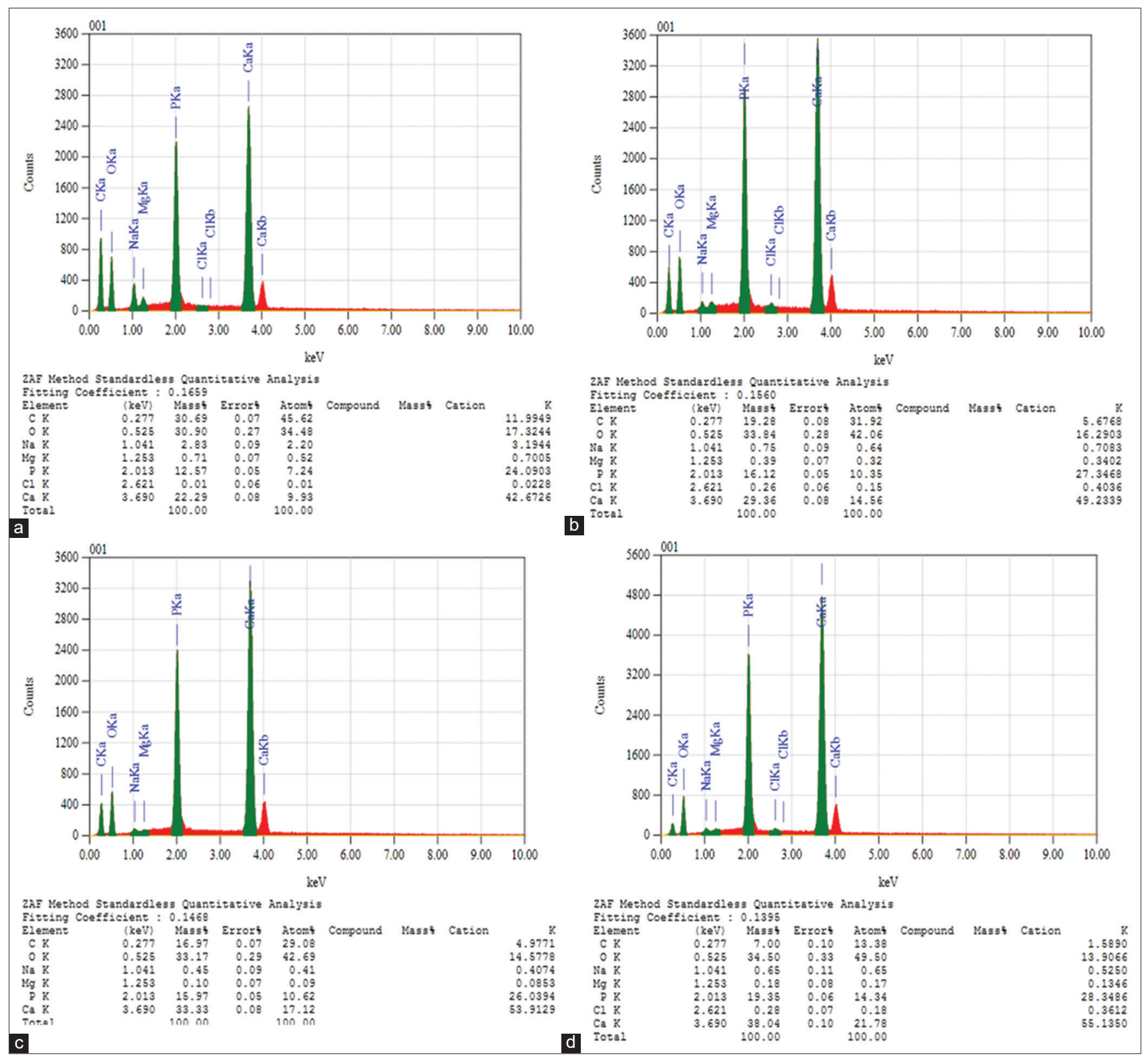

Fig. 2: Calcium and phosphate ions detected by energy-dispersive X-ray (EDX). (a) Demineralized dentin, (b) 3 days pAsp immersion, (c) 7 days pAsp immersion, (d) 14 days pAsp immersion

Table 2: The mean percentage of calcium and phosphate mineral levels in each group

\begin{tabular}{lll}
\hline Variable & Calcium (\%) & Phosphate (\%) \\
\hline Demineralized dentin & 27.1525 & 14.86 \\
3 days pAsp immersion & 27.005 & 14.9 \\
7 days pAsp immersion & 31 & 15.82 \\
14 days pAsp immersion & 26.8 & 13.96 \\
\hline
\end{tabular}

\section{CONCLUSION}

Remineralization began following 3 days of pAsp immersion that indicated by changes in dentinal tubule morphology after immersion in PILP containing pAsp. Remineralization occurred in the form of calcium and phosphate ion mineral deposits. Overall, these results indicate that including pAsp in the PILP process has the capability to rapidly remineralize demineralized dentin.

\section{ACKNOWLEDGMENTS}

This study was supported by the Directorate of Research and Community Engagement of Universitas Indonesia. The publication of this manuscript was supported by Universitas Indonesia.

\section{CONFLICTS OF INTEREST}

There are no conflicts of interest to declare.

\section{REFERENCES}

1. Habelitz LE, Marshall JH, Marshall GW Jr. Biomechanical perspective on the remineralization of dentin. Caries Res 2009;758:70-7.

2. Nudelman F, Lausch AJ, Sommerdijk NA, Sone ED. In vitro models of collagen biomineralization. J Struct Biol 2013;183:258-69.

3. Xiao S, Liang K, Weir MD, Cheng L, Liu H, Zhou X, et al. Combining bioactive multifunctional dental composite with PAMAM for root dentin remineralization. Materials 2017;10:1-17. 
4. Chen Z, Cao S, Wang H, Li Y, Kishen A, Deng X, et al. Biomimetic remineralization of demineralized dentine using scaffold of $\mathrm{CMC} / \mathrm{ACP}$ nanocomplexes in an in vitro tooth model of deep caries. PLoS One 2015;10:1-19.

5. Nudelman F, Pieterse K, George A, Bomans PH, Friedrich H, Brylka LJ, et al. The role of collagen in bone apatite formation in the presence of hydroxyapatite nucleation inhibitors. Nat Mater 2010;9:9-14.

6. Jee S, Thula TT, Gower LB. Acta biomaterialia development of bonelike composites via the polymer-induced liquid-precursor (PILP) process. Part 1 : Influence of polymer molecular weight. Acta Biomater 2010;6:3676-86

7. Gower LB. Biomimetic model systems for investigating the amorphous precursor pathway and its role in biomineralization. Chem Rev 2008:108:4551-627.

8. Saeki K, Chien Y, Nonomura G, Chin AF, Habelitz S, Gower LB, et al. Authors recovery after PILP remineralization of dentin lesions created with two cariogenic acids. Arch Oral Biol 2017;82:194-202.

9. Krogstad DV, Wang D, Lin-gibson S. Polyaspartic acid concentration controls the rate of calcium phosphate nanorod formation in high concentration systems. Biomacromolecules 2017;18:3106-13.

10. Nurrohman H, Habelitz S, Marshall GW, Gower LB. Influence of fluoride on the mineralization of collagen via the polymer-induced liquid-precursor (PILP) process. Dent Mater 2018;34:1378-90.

11. Burwell AK, Thula-mata T, Gower LB, Habelitz S, Kurylo M, Ho SP, et al. Functional remineralization of dentin lesions using polymerinduced liquid-precursor process. PLoS One 2012;7:e38852.

12. Dai L, Liu Y, Salameh Z, Khan S, Mao J, Pashley DH, et al. Can caries-affected dentin be completely remineralized by guided tissue remineralization? Dent Hypotheses 2011;2:74-82.

13. Xu Y, Koen CH, Arno PM. Microscopic structure of the polymerinduced liquid precursor for calcium carbonate microscopic structure of the polymer-induced liquid precursor for calcium carbonate. Nat Commun 2018:9:2582.

14. Bacino M, Girn V, Nurrohman H, Saeki K, Marshall SJ, Gower L, et al. Integrating the PILP-mineralization process into a restorative dental treatment. Dent Mater 2018;35:1-11. 